\title{
A Clinical Trial of the Accuracy and Treatment Experience of the Flash Glucose Monitor FreeStyle Libre in Adults with Type 1 Diabetes
}

\author{
Arndís F. Ólafsdóttir, $\mathrm{RN}, \mathrm{BSc}^{1,3}$ Stig Attvall, $\mathrm{MD}, \mathrm{PhD}_{1}^{2,3}$ Ulrika Sandgren, $\mathrm{RN}, \mathrm{BSc}^{2}$ \\ Sofia Dahlqvist, ${ }^{1}$ Aldina Pivodic, MSc, Stanko Skrtic, MD, PhD, MBA, \\ Elvar Theodorsson, and Marcus Lind, MD, $\mathrm{PhD}^{1,3}$
}

\begin{abstract}
Background: In Sweden, FreeStyle Libre a flash glucose monitoring system came onto the market in 2014 as a complement to self-monitoring of blood glucose. The aim of this study was to evaluate the accuracy and treatment experience of the FreeStyle Libre system.

Methods: Fifty-eight adults with type 1 diabetes used FreeStyle Libre for 10-14 days and measured capillary blood glucose levels with the HemoCue blood glucose measurement system at least six times a day simultaneously.

Results: For the entire study period, the mean absolute relative difference (MARD) was $13.2 \%(95 \%$ confidence interval [CI] $12.0 \%-14.4 \%$ ). MARD was $13.6 \%$ (95\% CI 12.1\%-15.4\%) during week 1 and $12.7 \%$ (95\% CI 11.5\%-13.9\%) during week 2. The mean absolute difference (MAD) for the whole study period was $19.8 \mathrm{mg} / \mathrm{dL}(1.1 \mathrm{mmol} / \mathrm{L})(95 \%$ CI $17.8-21.8 \mathrm{mg} / \mathrm{dL})$, including $20.5 \mathrm{mg} / \mathrm{dL}(1.14 \mathrm{mmol} / \mathrm{L})$ during week 1 and $19.0 \mathrm{mg} / \mathrm{dL}(1.05 \mathrm{mmol} / \mathrm{L})$ during week 2 . The overall correlation coefficient was 0.96 . For glucose values $<72,72-180$, and $>180 \mathrm{mg} / \mathrm{dL}(<4,4-10$, and $>10 \mathrm{mmol} / \mathrm{L})$, the MARD was $20.3 \%(95 \%$ CI $17.7 \%-23.1 \%$ ), $14.7 \%$ (95\% CI 13.4\%-16\%), and 9.6\% (95\% CI 8.5\%-10.8\%), respectively, and respective MAD values were $12.3,17.8$, and $23.6 \mathrm{mg} / \mathrm{dL}(0.69,0.99$, and $1.31 \mathrm{mmol} / \mathrm{L})$. Using the 10 -item visual analog scale, patients rated their experience with FreeStyle Libre as generally positive, with mean values ranging from 8.22 to 9.8 .

Conclusions: FreeStyle Libre had a similar overall MARD as continuous blood glucose monitoring systems in earlier studies when studied in similar at-home conditions. The overall patient satisfaction was high.
\end{abstract}

Keywords: Diabetes mellitus, Flash glucose monitor, Accuracy.

\section{Background}

N 2015 THERE WERE 415 million people with types 1 and 2 diabetes worldwide and this number is expected to increase to 642 million by 2040 with the highest prevalence of type 1 diabetes in Scandinavian countries. ${ }^{1,2}$ Good gly- cemic control is essential to reduce diabetic complications. ${ }^{3}$ Individuals with type 1 diabetes still have an excess risk of death compared with healthy individuals, which is strongly related to glycemic control. ${ }^{4,5}$

Frequent capillary blood glucose tests or self-monitoring of blood glucose $(\mathrm{SMBG})$ is the traditional and one of the

${ }^{1}$ Department of Medicine, NU Hospital Group, Uddevalla, Sweden.

${ }^{2}$ Department of Medicine, Sahlgrenska University Hospital, Gothenburg, Sweden.

${ }^{3}$ Department of Molecular and Clinical Medicine, University of Gothenburg, Gothenburg, Sweden.

${ }_{5}^{4}$ Statistiska Konsultgruppen, Gothenburg, Sweden.

${ }^{5}$ AstraZeneca R\&D, Mölndal, Sweden.

${ }^{6}$ Departments of Laboratory Medicine and Clinical and Experimental Medicine, Linköping University, Linköping, Sweden.

(C) Arndís F. Ólafsdóttir et al., 2017; Published by Mary Ann Liebert, Inc. This Open Access article is distributed under the terms of the Creative Commons Attribution Noncommercial License (http://creativecommons.org/licenses/by-nc/4.0/) which permits any noncommercial use, distribution, and reproduction in any medium, provided the original author(s) and the source are credited. 
most effective ways to keep track of individuals' blood glucose levels. ${ }^{3}$ It is recommended that individuals with type 1 diabetes test their blood glucose levels four to seven times a day for optimal control.6,7 During the recent decade, continuous glucose monitoring (CGM) has been shown to improve glycemic control and hemoglobin A1c (HbA1c); however, CGM is not widely available. ${ }^{8,9,10}$ In 2014, a new flash glucose monitoring (FGM) system was introduced. FGM consists of a subcutaneous sensor placed on the back of the upper arm, which measures glucose in the interstitial fluid every minute. Interstitial glucose concentrations can be monitored in real time by a hand-held reader placed over the sensor, which displays current and historical glucose levels, as well as trends over time. Unlike CGM, FGM does not sound a warning alarm when glucose levels are too high or too low and it does not need a capillary blood glucose sample for calibration, it is factory calibrated. FGM has a general lower cost than CGM as well as giving patients who do not use the CGM alarm function an alternative treatment. ${ }^{11}$ Currently, FGM is available in several European countries but not available in the United States.

The primary aim of this study was to evaluate the accuracy of the FreeStyle Libre FGM system (Abbott Diabetes Care, IL) for estimating plasma glucose levels in individuals with type 1 diabetes. A secondary aim was to evaluate the treatment experience of FreeStyle Libre.

\section{Method}

The study was carried out at two diabetes outpatient clinics in western Sweden, Uddevalla Hospital, part of the NUHospital group, and Sahlgrenska University Hospital. A nonrandomized, nonblinded, 14-day study was performed to determine the accuracy of the Abbott FreeStyle Libre FGM system in estimating blood glucose levels in individuals with type 1 diabetes. The study protocol was approved by the NUHospital group internal review board, Uddevalla and Trollhättan, Sweden.

Individuals of age 18-75 years with type 1 diabetes were included. Exclusion criteria consisted of history of allergic

Table 1. Inclusion and Exclusion CRiteria

Inclusion criteria
Type 1 diabetes
Adult patients, age 18 or older and $<75$ years
Written informed consent
Exclusion criteria
Pregnancy
Patients with severe cognitive dysfunction or other
disease that makes FGM use difficult
History of allergic reaction to any of the FGM system
materials or adhesives in contact with the skin
History of allergic reaction to chlorhexidine or alcohol
antiseptic solution
CGM or FGM usage in the past month
Abnormal skin at the anticipated glucose sensor
attachment sites (excessive hair, burn, inflammation,
infection, rash, and/or tattoo)

CGM, continuous blood glucose monitoring; FGM, flash glucose monitoring. reaction to any FGM materials or adhesives in contact with the skin, history of allergic reaction to chlorhexidine or alcohol antiseptic solution, CGM or FGM usage in the last month, and abnormal skin at the anticipated glucose sensor attachment sites (excessive hair, burn, inflammation, infection, rash, and/or tattoo) (Table 1).

After obtaining written and verbal informed consent, a diabetes nurse demonstrated how to insert the sensor that was inserted during the visit. The study period lasted 14 days, which is the length of time the sensor is active. Information on the FreeStyle Libre screen was not masked. Each participant received a new FreeStyle Libre. Individuals were instructed on how to use FreeStyle Libre, and how to perform the SMBG measurements with a HemoCue blood glucose meter. We used HemoCue DM RT 201. All HemoCue meters were calibrated before they were used in the study using an absolute isotop dilution GC-MC measurement system. ${ }^{12}$ The total measurement error/reproducibility imprecision of HemoCue is less than $6.5 \% .^{13}$ In earlier studies using both capillary and venous values as a reference when evaluating CGM accuracy, there was a strong correlation between venous and capillary HemoCue concentrations, and capillary concentrations were shown to be a suitable reference. ${ }^{14}$

Participants documented the blood glucose concentrations measured using HemoCue and simultaneously recorded FreeStyle Libre levels in diaries. Participants were instructed to only dose insulin based on SMBG measurements. Participants were expected to measure at least six SMBG concentrations using HemoCue per day and the same time document a FreeStyle Libre value. After 14 days, the participants returned both devices. Site personnel verified the diaries by going through all values registered in the HemoCue meters and FreeStyle Libre. The data from FreeStyle Libre were downloaded using the FreeStyle Libre computer software. If the sensor became dislodged within the first 10 days, a new sensor was inserted and the participant started again from day 1 . At a subsequent study visit, participants completed the 10-item visual analog scale (VAS) to rate their experience of FreeStyle Libre, similar to questionnaires used earlier in evaluations of CGM systems. ${ }^{15}$ Questionnaires were filled in under calm and quiet circumstances. The insertion site was examined after the sensor was removed for inflammation, bleeding, infection, or other skin reaction.

\section{End points}

The key end points were predefined and registered on ClinicalTrials.gov: NCT02677454. The primary end point was the mean absolute relative difference (MARD) of all estimated capillary glucose values registered during the whole study period between the FreeStyle Libre system and the capillary blood glucose concentration measured with HemoCue. Secondary end points were mean absolute difference (MAD) of all estimated capillary glucose values registered during the whole study period of the FreeStyle Libre system compared with the HemoCue system. The Pearson correlation coefficient between FreeStyle Libre and the capillary reference blood glucose measured with HemoCue was also estimated. The MARD, MAD, and Pearsons correlation were also analyzed during days 1-7 and days 8-14 
and in the following specified glucose ranges: $<72 \mathrm{mg} / \mathrm{dL}$ ( $4 \mathrm{mmol} / \mathrm{L}), 72-180 \mathrm{mg} / \mathrm{dL}(4-10 \mathrm{mmol} / \mathrm{L})$, and $>180 \mathrm{mg} / \mathrm{dL}$ $(10 \mathrm{mmol} / \mathrm{L})$. VAS questionnaires consisting of 10 statements were administered to evaluate participant experiences with FreeStyle Libre.

\section{Independence of the study}

The manufacturer of FreeStyle Libre was not involved in the design or conduct of the study, data analysis, interpretation of results, or publication of the article. No financial or in-kind support was received from the manufacturer for systems, sensors, salaries, or other costs.

\section{Statistics}

For a $95 \%$ confidence interval (CI) for MARD using FreeStyle Libre with a range of no more than $\pm 1.5 \%$ assuming a standard deviation of $5.5 \%$ (from a previous study of 38 patients using the DexCom G4 and Medtronic Enlite CGM sensors ${ }^{15}$ ), 52 subjects were required to be enrolled in this study.

All statistical analyses were predefined in the statistical analysis plan before database lock. All participants having at least 10 time points with evaluable blood glucose values from FreeStyle Libre and the reference capillary value obtained from HemoCue during the whole study period were included in the intention-to-treat population. All matching time points were used.

For descriptive purposes, mean, standard deviation, median, minimum, and maximum values are presented for continuous variables, and number and percentages are presented for categorical variables. Bootstrapped 95\% CIs (with 10,000 replicates) were obtained for predefined accuracy variables.

The primary variable was MARD, which is the absolute relative difference between estimated capillary glucose values of FreeStyle Libre and blood glucose measured with HemoCue: $\left(\left|\mathrm{FGM}_{\mathrm{i}}-\mathrm{HemoCue}_{\mathrm{i}}\right|\right) / \mathrm{HemoCue}_{\mathrm{i}}$, where $\mathrm{i}$ is time point in the study.

The primary end point and other continuous variables were tested using the Wilcoxon signed rank test. The relation between two continuous variables was expressed by the Pearson correlation coefficient. For graphical purposes, the relation between MARD and MAD versus HemoCue concentrations was investigated using mixed models, taking intraindividual correlations into account. Random effects and residual covariance structure were determined using Akaike information criterion.

All tests were two tailed and conducted at the 0.05 significance level. Analyses were performed using SAS version 9.4 (SAS Institute, Inc., Cary, NC).

\section{Results}

Fifty eight individuals participated in the study, of which 50 had at least 10 time points from FreeStyle Libre and HemoCue pairs of matched glucose measurements. Two patients received faulty HemoCue meters, two patients discontinued the study, three patients did not have enough SMBG measurements, and one patient was lost to followup. Of the 50 participants who met the analysis criteria, $40 \%$ were women, mean age was 47 years, mean HbAlc was $8.2 \%(65.9 \mathrm{mmol} / \mathrm{mol}) \mathrm{min}-\mathrm{max} \mathrm{HbA} 1 \mathrm{c}$ was $5.5 \%-12.6 \%$
Table 2. Demographics and Baseline Characteristics (Intention-to-Treat Population)

\begin{tabular}{|c|c|}
\hline Variable & $\operatorname{ITT}(\mathrm{n}=50)$ \\
\hline Age & $\begin{array}{c}47.2(15.7) \\
50.0(19.0 ; 77.0) \\
n=50\end{array}$ \\
\hline \multicolumn{2}{|l|}{ Sex } \\
\hline Male & $30(60.0 \%)$ \\
\hline Female & $20(40.0 \%)$ \\
\hline Diabetes duration & $\begin{array}{c}21.4(13.1) \\
16.8(1.1 ; 60.7) \\
n=50\end{array}$ \\
\hline BMI $\left(\mathrm{kg} / \mathrm{m}^{2}\right)$ & $\begin{array}{c}25.5(4.2) \\
24.7(19.3 ; 35.9) \\
n=49\end{array}$ \\
\hline Smoking & $8(16.0 \%)$ \\
\hline HbA1c (\%, NGSP) & $\begin{array}{c}8.18(1.40) \\
8.10(5.54 ; 12.58) \\
n=50\end{array}$ \\
\hline $\mathrm{A} / \mathrm{C}$ ratio & $\begin{array}{c}2.02(4.09) \\
0.90(0.20 ; 20.90) \\
n=43\end{array}$ \\
\hline \multicolumn{2}{|l|}{ Insulin delivery } \\
\hline CSII & $13(26.0 \%)$ \\
\hline MDI & $37(74.0 \%)$ \\
\hline Insulin dose & $\begin{array}{c}47.8(25.0) \\
40.0(15.0 ; 136.0) \\
n=50\end{array}$ \\
\hline Systolic blood pressure & $\begin{array}{c}128.7(14.6) \\
128.0(104.0 ; 166.0) \\
n=49\end{array}$ \\
\hline Diastolic blood pressure & $\begin{array}{c}71.4(9.3) \\
72.0(43.0 ; 93.0) \\
n=49\end{array}$ \\
\hline $\begin{array}{l}\text { Average number of hypoglycemia } \\
\text { per month }\end{array}$ & $\begin{array}{c}6.64(6.99) \\
4.00(0.00 ; 30.00) \\
n=50\end{array}$ \\
\hline Previous myocardial infarction & $0(0.0 \%)$ \\
\hline Previous stroke & $1(2.0 \%)$ \\
\hline Previous amputation & $1(2.0 \%)$ \\
\hline Previous foot ulcers & $0(0.0 \%)$ \\
\hline Current foot ulcers & $4(8.0 \%)$ \\
\hline Previous retinopathy & $3(6.0 \%)$ \\
\hline
\end{tabular}

For categorical variables $n(\%)$ is presented.

For continuous variables mean (SD)/median (Min; Max) $/ n$ is presented.

$\mathrm{A} / \mathrm{C}$, albumin/creatinine; CSII, continuous subcutaneous insulin infusion; HbA1c, hemoglobin A1c; ITT, intention-to-treat; MDI, multiple daily insulin injections; NGSP, National Glycohemoglobin Standardization Program.

(37-114 mmol/mol), and mean diabetes duration was 21 years (Table 2).

\section{End points of accuracy evaluations}

The MARD for the whole study period was $13.2 \%$ (95\% CI 12.0\%-14.4\%.) and the MAD was $19.8 \mathrm{mg} / \mathrm{dL}$ $(1.10 \mathrm{mmol} / \mathrm{L})(95 \% \mathrm{CI} 17.8-21.8 \mathrm{mg} / \mathrm{dL})$. When comparing the results of week 1 with those of week 2 , neither the 


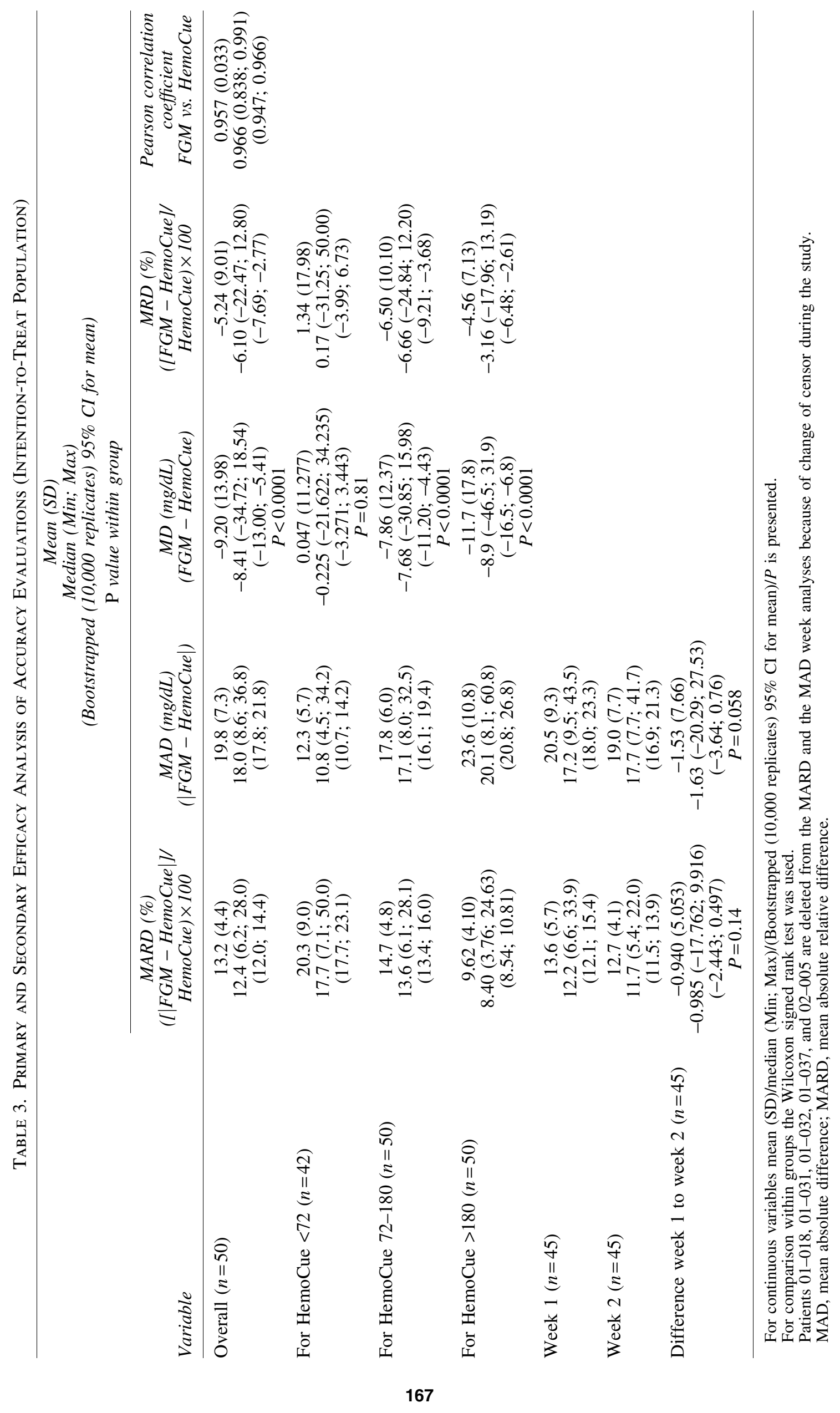



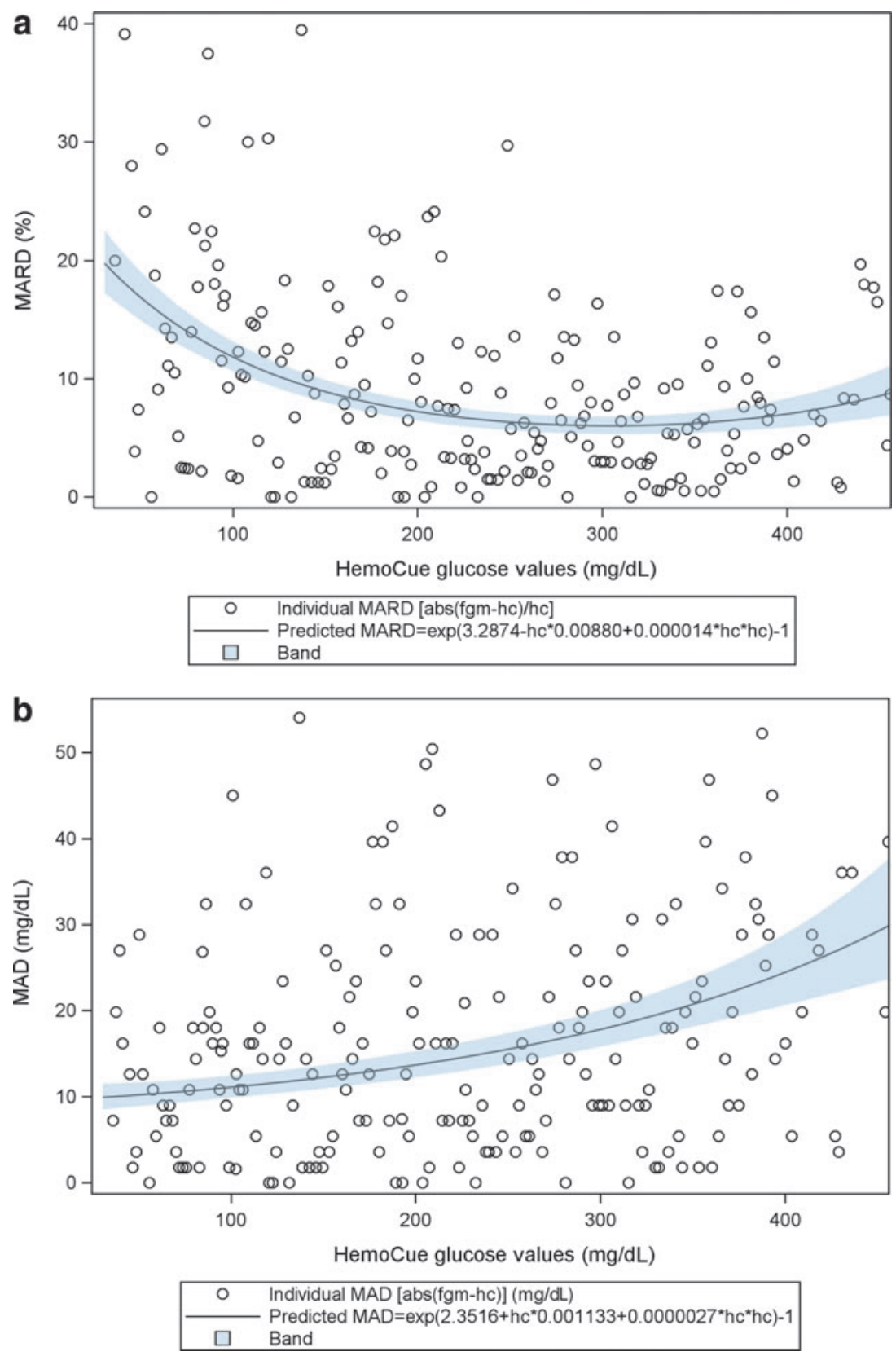

FIG. 1. (a) The MARD of FGM (FreeStyle Libre) in relation to glucose levels measured by HemoCue as an exponentiated quadratic function of glucose levels measured by HemoCue. Figure shows each single measurement as well as the predicted MARD with $95 \%$ CI. (b) The MAD of FGM (FreeStyle Libre) in relation to glucose levels measured by HemoCue as an exponentiated quadratic function of glucose levels measured by HemoCue. Figure shows each single measurement as well as the predicted MAD with $95 \%$ CI. (c) Scatter plot of measurements of glucose levels by FGM (FreeStyle Libre) versus HemoCue. The red line represents for one-one relation. The figure shows a greater concentration of values below the red line, indicating lower mean glucose levels by FGM than mean glucose levels obtained by HemoCue. CI, confidence interval; FGM, flash glucose monitoring; MAD, mean absolute difference; MARD, mean absolute relative difference.

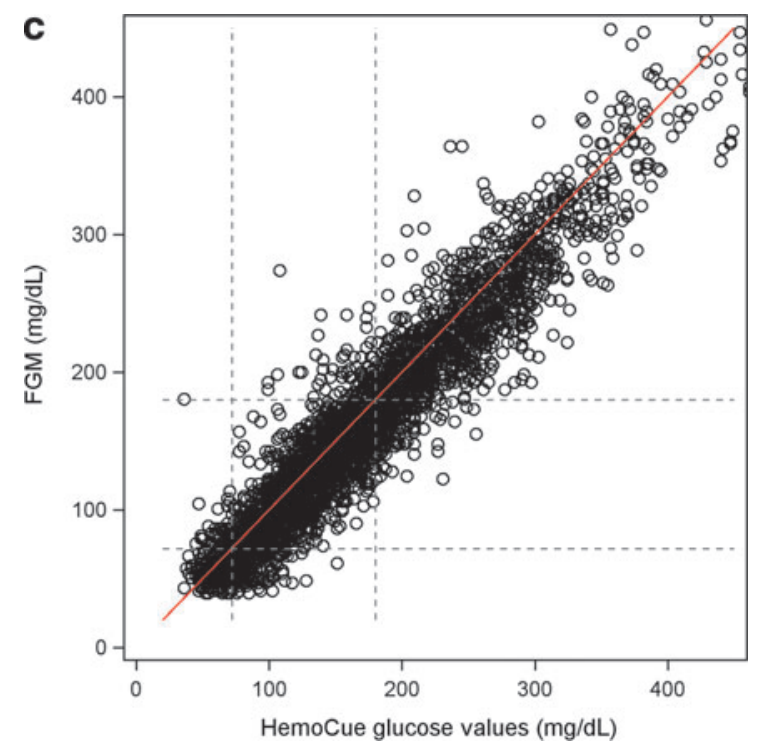


MARD nor the MAD changed significantly. The MARD decreased by $-0.9 \%$ and the MAD decreased by $-1.53 \mathrm{mg} / \mathrm{dL}$ $(0.09 \mathrm{mmol} / \mathrm{L})$ in week 2 as compared with those in week 1 (Table 3).

The MARD decreased with higher blood glucose values with the MARD at $20 \%$ for blood glucose values $<72 \mathrm{mg} / \mathrm{dL}(4 \mathrm{mmol} / \mathrm{L})$ and at $9.6 \%$ for blood glucose values $>180 \mathrm{mg} / \mathrm{dL}(10 \mathrm{mmol} / \mathrm{L})$. The MAD in contrast increased with higher blood glucose values with the MAD of $12.3 \mathrm{mg} / \mathrm{dL}(0.69 \mathrm{mmol} / \mathrm{L})$ for glucose values $<72 \mathrm{mg} / \mathrm{dL}(4 \mathrm{mmol} / \mathrm{L})$ and $23.6 \mathrm{mg} / \mathrm{dL}(1.31 \mathrm{mmol} / \mathrm{L})$ for glucose values $>180 \mathrm{mg} / \mathrm{dL}(10 \mathrm{mmol} / \mathrm{L})$ (Table 3 and Fig. 1a, b).
The Pearson correlation coefficient between glucose values measured by HemoCue and FreeStyle Libre for the whole study period was 0.96 (95\% CI 0.95-0.97).

\section{Treatment experience}

The participants rated their experience with FreeStyle Libre using the 10-statement VAS (0-10) as positive with mean values ranging from 8.22 to 9.8 . For the statement "My experience of FreeStyle Libre was very positive," the mean was 9.04. For the statement "It was easy to use FreeStyle Libre," the mean was 9.8. The mean number of scanning problems with FreeStyle Libre was 0.48 (95\% CI 0.22-0.78).

Table 4. End Points of Patient Treatment Experience (Intention-to-Treat Population)

Variable

$\operatorname{ITT}(\mathrm{n}=50)$

Questionnaire (VAS)

My experience of Freestyle Libre was very positive (0-10)

$9.04(1.51)$

$10.00(3.00 ; 10.00)$ $n=50$

The insertion of Freestyle Libre was very easy and trouble free (0-10)

$9.08(1.79)$

$10.00(0.00 ; 10.00)$ $n=50$

During my time using Freestyle Libre, I felt safe and free (0-10)

$8.92(1.34)$

$9.00(5.00 ; 10.00)$ $n=50$

It was easy to use Freestyle Libre (0-10)

$9.80(0.64)$

$10.00(6.00 ; 10.00)$ $n=50$

It was easy to interpret the information on the Freestyle Libre screen $(0-10)$

$9.64(0.85)$

$10.00(6.00 ; 10.00)$ $n=50$

I was not in pain or had discomfort in connection to my use of Freestyle Libre (0-10)

I experienced no problem scanning Freestyle Libre (0-10)

$9.06(2.03)$

$10.00(0.00 ; 10.00)$ $n=50$

$9.70(0.71)$

$10.00(7.00 ; 10.00)$ $n=50$

The Freestyle Libre sensor was comfortable to have on my body

$8.32(2.26)$

in my daily life (0-10)

$9.00(0.00 ; 10.00)$ $n=50$

The Freestyle Libre sensor did not disturb my daily life (e.g., how I choose to dress, my work, or other daily activities) (0-10)

$8.22(2.26)$

$9.00(0.00 ; 10.00)$ $n=50$

I would like to use Freestyle Libre in my daily life (0-10)

$9.40(1.56)$

$10.00(3.00 ; 10.00)$ $n=50$

Have you at some point tried a CGM (yes, no)

No

$34(69.4 \%)$

Yes

$15(30.6 \%)$

Secretion of blood and other fluids at removal (yes, no)

No

$41(87.2 \%)$

Yes

$6(12.8 \%)$

Visible skin reaction after removal (yes, no)

No

$32(68.1 \%)$

Yes

$15(31.9 \%)$

For categorical variables $n(\%)$ is presented.

For continuous variables mean (SD)/median (Min; Max) $/ n$ is presented.

VAS, visual analog scale. 


\section{Skin reactions at sensor use}

Of all patients, $6(13 \%)$ had blood and other fluids at the sensor site when the sensor was removed and $15(32 \%)$ had a visible skin reaction after removal (Table 4).

\section{Post hoc analyses}

Of all analyzed glucose concentrations, $80.1 \%$ (2859 out of 3602 ) deviated less than or equal to $20 \%$. The corresponding percentages for blood glucose concentrations $<72$, $72-180$, and $>180 \mathrm{mg} / \mathrm{dL}(4,4-10$, and $>10 \mathrm{mmol} / \mathrm{L})$ were $63.4 \%, 74.6 \%$, and $91.3 \%$, respectively. Of all analyzed glucose concentrations, $92.1 \%$ deviated less than $30 \%$ and for the three glucose categories it was $81.7 \%, 89.5 \%$, and $97.9 \%$, respectively.

The difference between the mean glucose values measured by HemoCue and FreeStyle Libre over the entire study period was $-9.20 \mathrm{mg} / \mathrm{dL}$ (95\% CI -13.0 to -5.4$),-0.51 \mathrm{mmol} / \mathrm{L}$ (95\% CI -0.72 to -0.3$) P<0.0001$. The mean difference for glucose values $72-180 \mathrm{mg} / \mathrm{dL}$ (4-10 $\mathrm{mmol} / \mathrm{L}$ ) was $-7.86 \mathrm{mg} / \mathrm{dL}$ $(-0.436 \mathrm{mmol} / \mathrm{L})$ and that for glucose value of $>180 \mathrm{mg} / \mathrm{dL}$ $(10 \mathrm{mmol} / \mathrm{L})$ was $-11.7 \mathrm{mg} / \mathrm{dL}(-0.65 \mathrm{mmol} / \mathrm{L})$, both were found to be significant, $P<0.0001$. However, the mean difference for glucose values $<72 \mathrm{mg} / \mathrm{dL}(4 \mathrm{mmol} / \mathrm{L})$ was 0.047 $\mathrm{mg} / \mathrm{dL}(0.003 \mathrm{mmol} / \mathrm{L})$, which was not significant, $P=0.81$.

\section{Discussion}

In this study, the FreeStyle Libre FGM was associated with a $13.2 \%$ MARD as compared with capillary blood glucose values measured with the HemoCue device after 2 weeks of use. At the end of week 1, the MARD was $13.6 \%$, and decreased only slightly to $12.7 \%$ at the end of week 2 . The MAD in glucose levels measured between FreeStyle Libre and HemoCue reference over the whole study period was $19.8 \mathrm{mg} / \mathrm{dL}(1.1 \mathrm{mmol} / \mathrm{L})$. The FreeStyle Libre system showed glucose values that were significantly lower than those of HemoCue at $-9.20 \mathrm{mg} / \mathrm{dL}$ $(-0.51 \mathrm{mmol} / \mathrm{L})$

We found $19.9 \%$ of glucose values measured by FreeStyle Libre deviated more than $20 \%$, and $7.9 \%$ of glucose values measured by FreeStyle Libre deviated more than $30 \%$ from the HemoCue reference. The participants rated their overall treatment experience with FreeStyle Libre as high.

Using a similar study design, we previously studied the accuracy and treatment experience of the DexCom G4 and Enlite CGM systems (using portable systems not linked to continuous subcutaneous insulin infusion [CSII]). ${ }^{15}$ The absolute majority of reference values in our work were also measured in the ambulatory setting using the HemoCue meter. Although comparisons between devices not tested "head to head" in the same trial should be cautiously interpreted, it is noteworthy that the MARD in this study was similar overall to that of the DexCom G4 system and lower than that of the Enlite sensor in our previous work $(13.2 \%$, $13.9 \%$, and $17.8 \%$, respectively). ${ }^{15}$ However, novel CGM sensors and algorithms are under development, and therefore accuracy may differ with respect to earlier studies using sensors linked to an insulin pump or used with more novel portable systems.

A similar study, which compared FreeStyle Libre against both its own built-in capillary blood glucose meter and yellow spring instrument, found the MARD was $12.1 \%$ and
$12 \%$, respectively. ${ }^{16}$ In the Yellow Spring study, each patient received two FreeStyle Libre sensors simultaneously compared with only one sensor used in our study. In our study, the correlation between HemoCue and FreeStyle Libre was strong at 0.957 with FreeStyle Libre in most cases showing lower concentrations than HemoCue (Fig. 1c). It is possible the MARD may have been somewhat lower if there had not been any systematic deviation between the Freestyle Libre and HemoCue values. The measurement results of both FreeStyle Libre and HemoCue are traceable to measurement results using primary reference materials from the National Institute of Standards and Technologies (NIST). HemoCue uses isotope dilutation mass spectrometry for glucose and primary reference material from NIST to simultaneously measure at least 20 fresh patient samples from healthy and individuals with diabetes to calibrate each cuvette lot. The bias shown in our results between FreeStyle Libre and HemoCue is likely because of the use of noncommutable reference materials and calibrators in a multistep traceability chain in the case of FreeStyle Libre. ${ }^{17-20}$ This negative bias could possibly be adjusted with adjusted algorithms.

There are several implications regarding the accuracy of FreeStyle Libre in this study. First, it shows that FreeStyle Libre has an overall accuracy that is good, especially considering that it measures glucose concentrations in the extracellular fluid and does not need to be calibrated. However, our study shows that FreeStyle Libre measures systematically lower glucose concentrations (has a negative bias) than the HemoCue whole capillary blood method. This deviation has also been noted by many patients in clinical practice in Sweden where the study was carried out. A non-negligible proportion of glucose concentrations measured using FreeStyle Libre also deviated more than $20 \%$ and $30 \%(19.9 \%$ and $7.9 \%$ of values, respectively). As the system is approved in Europe to dose insulin according to measured glucose values, these findings warrant some caution, particularly in specific situations in which the glucose level is of particular clinical importance for the patient or the healthcare official. Deviation of more than $20 \%$ and especially more than $30 \%$ can likely be crucial in several clinical situations. However, a recent randomized study limited to patients with relatively good glycemic control (HbA1c $<7.5 \%)$ using FGM found a $38 \%$ reduction in patient time in hypoglycemia compared with controls, indicating safety from a hypoglycemia perspective in this patient group. ${ }^{21}$ It is, therefore, possible that the lower accuracy of the Libre meter than the conventional SMBG meters is compensated by patients making more glucose checks and receiving information of glucose trends. Long-term trials of FGM and in a more general population of persons with type 1 diabetes are further needed,

Using the 10-item VAS questionnaire, participants rated their experience with FreeStyle Libre as positive, as has been shown in earlier studies, ${ }^{16,21}$ with average scores from 8.22 to 9.8 on a scale from 0 to 10 . Along with accuracy comparisons, indirect comparisons should be interpreted cautiously, but it is noteworthy that average scores from earlier studies in which patients rated the DexCom G4 system using the same questions ranged from 72.5 to 90 (out of 100) and average scores for the Enlite sensor in conjunction to the Guardian system ranged from 42.1 to 86.1 (out of 100). ${ }^{15}$ In the earlier study, patients also wore the Dexcom and Guardian systems 
simultaneously, thus treatment experience was evaluated under somewhat different circumstances.

The introduction of FreeStyle Libre offers patients a new alternative for managing their blood glucose concentration, which seems to be equally accurate to CGM and requires less daily effort on the patient's part in performing the recommended amount of SMBG. ${ }^{6,7,15}$ Earlier studies have shown a reduction in HbA1c with CGM use in conjunction with insulin pump treatment and multiple daily insulin injections. ${ }^{8,9,10}$ but there are no data from randomized studies currently available regarding the effect of FGM on HbA1c. We can speculate that FGM will give patients who find it burdening to take SMBG an improved possibility to lower their HbA1c. Furthermore, it is noteworthy that FGM and CGM differ. Namely, FGM does not sound an alarm with hyperglycemia and hypoglycemia. However, potential differences with respect to effects on glycemic control, hypoglycemia risk, and other safety concerns between FGM and CGM are currently not known and would need investigations in randomized clinical trials.

The strength of this study is that it was performed fully independently of the FreeStyle Libre manufacturer. All costs of FreeStyle Libre as well as salaries paid to research staff were supplied by independent funds. The manufacturer of Freestyle Libre was not involved in any parts of the trial. The key end points for the study were predefined before the study began. Owing to the fact that the study was carried out at home, there was an increased possibility to capture hypoglycemic values for analysis and the questionnaire that captured subject's experience of the system is also a strength of this study. Limitations include the short study duration. Glucose values were measured by patients themselves, thus testing procedures were not controlled, although all patients received careful instructions of these procedures. The results of the questionnaire should be viewed with some cautiousness because it has not been structurally validated and treatment experience can be difficult to fully evaluate over a relative short time period.

In conclusion, the FreeStyle Libre system seems to have an overall accuracy that is similar to CGM-systems with high accuracy. The treatment experience was high. A nonnegligible proportion of glucose values, however, deviates more than $20 \%$ and $30 \%$, which is of concern to be aware of in clinical practice when dosing insulin. The calibration of the FreeStyle Libre system could likely be improved because it had a negative bias compared with HemoCue capillary whole blood.

\section{Acknowledgments}

This study was performed independently of the manufacturers of FreeStyle Libre and HemoCue. No salaries, materials, or other study-related support were received from these manufacturers. Support for the study was received from the Novo Nordisk Foundation; the Swedish state, under an agreement between the Swedish national government and the county councils concerning economic support for research and education of doctors (ALF-agreement); Health \& Medical Care Committee of the regional Executive Board, Region Vastra Gotaland, Sweden; and the Fyrbodal Research and Development Council, Region Vastra Gotaland, Sweden.

\section{Author Disclosure Statement}

M.L. has received honoraria and/or consulting fees from Astra Zeneca, Eli Lilly, Medtronic, and Novo Nordisk; research grants from Astra Zeneca, Dexcom, and Novo Nordisk; and has participated on advisory boards for Novo Nordisk. E.T. is member of the board of the company MediRox that develops and markets reagents for diagnosing blood coagulation disorders. S.S. is an employee of AstraZeneca. A.F.O., S.A., S.D., U.S., and A.P. declare no conflict of interests.

\section{References}

1. International Diabetes Federation: IDF Diabetes Atlas, 7th ed. Brussels: International Diabetes Federation (IDF), 2015.

2. Hanås R: Type 1 Diabetes in Children, Adolescents and Young Adults. 6th ed. Uddevalla: BetaMed; 2015.

3. Diabetes Control and Complications Trial Study Group: The effect of intensive treatment of diabetes on the development and progression of long-term complications in insulin-dependent diabetes mellitus. N Engl J Med 1993; 329:977-986.

4. Ahlén E, Pivodic A, Wedel H, et al.: Glycemic control, renal complications, and current smoking in relation to excess risk of mortality in persons with type 1 diabetes. J Diabetes Sci Technol 2016;10:1006-1014.

5. Lind M, Svensson AM, Kosiborod M, et al.: Glycemic control and excess mortality in type 1 diabetes. $\mathrm{N}$ Engl $\mathrm{J}$ Med 2014;371:1972-1982.

6. American Diabetes Association. Standards of medical care for patients with diabetes mellitus. Diabetes Care 2003;26 Suppl 1:S33-S50.

7. Attvall S. Egenmätning av blodglukos vid diabetesregional Medicinsk Riktlinje-Läkemedel. 2016; https://alfresco .vgregion.se/alfresco/service/vgr/storage/node/content/3603/ Egenm\%C3\%A4tning\%20av\%20blodglukos\%20vid\%20 diabetes.pdf?a=false \&guest=true (accessed February 9, 2017).

8. Lind M, Polonsky W, Hirsch IB, et al.: Continuous glucose monitoring vs conventional therapy for Glycemic Control in adults with type 1 diabetes treated with multiple daily insulin Injections: The GOLD Randomized Clinical Trial. JAMA 2017;317:379-387.

9. Battelino T, Conget I, Olsen B, et al.: The use and efficacy of continuous glucose monitoring in type 1 diabetes treated with insulin pump therapy: a randomised controlled trial. Diabetologia 2012;55:3155-3162.

10. Beck RW, Riddlesworth T, Ruedy K, et al.: Effect of continuous glucose monitoring on glycemic control in adults with type 1 diabetes using insulin injections: The DIAMOND Randomized Clinical Trial. JAMA 2017;317:371-378.

11. Produkter CL: Sortimentskatalog 2015 diabeteshjälpmedel. 2015; http://www.vgregion.se/upload/Regionservice/CLP/ Diabetes/Sortimentskatalog\%20Diabetes\%202017.pdf (accessed February 9, 2017).

12. Hannestad U, Lundblad A: Accurate and precise isotope dilution mass spectrometry method for determining glucose in whole blood. Clin Chem 1997;43:794-800.

13. Kos S, van Meerkerk A, van der Linden J, et al.: Validation of a new generation POCT glucose device with emphasis on aspects important for glycemic control in the hospital care. Clin Chem Lab Med 2012;50:1573-1580.

14. Andelin M, Kropff J, Matuleviciene V, et al.: Assessing the accuracy of continuous glucose monitoring (CGM) calibrated with capillary values using capillary or venous 
glucose levels as a reference. J Diabetes Sci Technol 2016; 10:876-884.

15. Matuleviciene V, Joseph JI, Andelin M, et al.: A clinical trial of the accuracy and treatment experience of the Dexcom G4 sensor (Dexcom G4 system) and Enlite sensor (guardian REAL-time system) tested simultaneously in ambulatory patients with type 1 diabetes. Diabetes Technol Ther 2014;16:759-767.

16. Bailey T, Bode BW, Christiansen MP, et al.: The performance and usability of a factory-calibrated flash glucose monitoring system. Diabetes Technol Ther 2015;17: 787-794.

17. Armbruster D: Measurement traceability and US IVD manufacturers: the impact of metrology. Accredit Qual Assur 2009;14:393-398.

18. Bièvre PD, Kaarls R, Peiser HS, et al.: Measurement principles for traceability in chemical analysis. Accredit Quality Assurance 1996;1:3.
19. Bièvre PD, Kaarls R, Peiser HS, et al.: Protocols for traceability in chemical analysis. Accredit Qual Assur 1997;2:270.

20. Bièvre $P$, Kaarls R, Peiser HS, et al.: Protocols for traceability in chemical analysis. Accredit Qual Assur 1997;2:168.

21. Bolinder J, Antuna R, Geelhoed-Duijvestijn P, et al.: Novel glucose-sensing technology and hypoglycaemia in type 1 diabetes: a multicentre, non-masked, randomised controlled trial. Lancet 2016;388:2254-2263.

Address correspondence to: Arndis F. Ólafsdóttir, RN, BSc

Diabetesmottagningen Uddevalla Sjukhus Fjällvägen 9 Uddevalla 45180 Sweden

E-mail: finna.olafsdottir@vgregion.se 